\title{
AMBER and MIDI interferometric observations of the post-AGB binary IRAS 08544-4431: the circumbinary disc resolved ${ }^{\star}$
}

\author{
P. Deroo ${ }^{1}$, B. Acke ${ }^{1, \star \star}$, T. Verhoelst ${ }^{1, \star \star}$, C. Dominik ${ }^{2}$, E. Tatulli $^{3}$, and H. Van Winckel ${ }^{1}$ \\ 1 Instituut voor Sterrenkunde, KU Leuven, Celestijnenlaan 200D, 3001 Leuven, Belgium \\ e-mail: pieter.deroo@ster.kuleuven.be \\ 2 Astronomical Institute "Anton Pannekoek", University of Amsterdam, Kruislaan 403, 1098 SJ Amsterdam, The Netherlands \\ 3 Observatorio di Arcetri, Instituto Nazionale di Astrofisica, Large E. Fermi 5, 50125 Firenze, Italy
}

Received 13 June 2007 / Accepted 18 September 2007

ABSTRACT

\begin{abstract}
Context. For a large group of post-AGB binaries, the presence of a stable reservoir of dust is postulated. Although this reservoir will influence the final evolution stages of these objects significantly, its actual geometry and structure remains largely unknown.

Aims. We aim at determining the dust morphology of a member of this group, IRAS 08544-4431.

Methods. We use the interferometric capabilities of the AMBER and MIDI instruments, operating in the K and N-band respectively. The high spatial resolution measurements are used in conjunction with the broad band spectral characteristics to determine the dust geometry, based on self consistent $2 \mathrm{D}$ radiative transfer models.

Results. We resolve the object in both K and N. Moreover, using the closure phase capabilities of AMBER, we measure in the K-band a large asymmetry of the dusty environment. The interferometric data are clearly incompatible with a spherical outflow. We model the dusty environment with a passive irradiated dusty disc model. Although this model is constrained mainly on the basis of the spectral energy distribution, it reproduces simultaneously the amplitude and closure phase of the visibilities, in both wavelength bands.

Conclusions. Our model of a passive, irradiated disc in equilibrium gives an excellent fit to both the $\mathrm{K}$ and $\mathrm{N}$-band visibilities and closure phase. The dust around this evolved binary star is indeed locked in a circumbinary disc with a significant scale height. Grain growth, settling, radial mixing and crystallization are efficient in such an environment. We conclude that the circumbinary disc of this evolved object, is governed by the same physical processes that govern the proto-planetary discs around young stellar objects.
\end{abstract}

Key words. techniques: interferometric - stars: AGB and post-AGB - stars: circumstellar matter -

stars: individual: IRAS 08544-4431

\section{Introduction}

The final evolution of low- and intermediate-mass stars is a rapid transition from the Asymptotic Giant Branch (AGB) over the post-AGB phase towards the Planetary Nebula phase (PNe), before the stellar remnant cools down as a White Dwarf (WD). The processes leading to PNe formation are complex and poorly known (e.g. Balick \& Frank 2002, and references therein) and arguably the biggest challenge lies in understanding the origin of the remarkable morphological and kinematical differences between AGB circumstellar envelopes and the more evolved counterparts. The debate on which physical mechanisms are driving the morphology changes has gained even more impetus with the finding that resolved proto-planetary nebulae (PPNe) display a surprisingly wide variety in shape and structure, very early in their evolution off the AGB (e.g. Sahai et al. 2007, and references therein). A heavily debated topic in the recent international $\mathrm{PNe}$ conferences was the growing evidence that the physics of Planetary Nebulae creation may be dominated by processes relevant only in binary stars (e.g. Moe \& De Marco 2006; Zijlstra 2007).

Testing of the binary hypothesis is severely hampered by the lack of direct observational information on binarity in PNe and

\footnotetext{
* Based on observations collected at the European Southern Observatory, Chile: ESO.076.D-0274, ESO.078.D-0113.

$\star \star$ Postdoctoral Fellows of the Fund for Scientific Research, Flanders.
}

PPNe, in combination with our poor theoretical understanding of the possible binary interaction processes and their impact on the shape and shaping of the circumstellar environment.

To study late stellar evolution in binary systems, optically bright, less obscured post-AGB stars are ideal candidates and in recent years it became clear that binarity is a rather widespread phenomenon (Van Winckel 2003). In most sample stars, dust is found near sublimation temperature, despite the observed intermediate spectral types of the central stars which are not likely to be in an active dust production phase.

Most objects of this sample are very distant, up to several kpc away. With the advent of near- and mid-infrared (spectro-) interferometric instrumentation, we have now the unique capability to probe directly these suspected very compact circumstellar structures.

In this Letter we report on our results for IRAS 08544-4431 (V390 Vel, hereafter IRAS 08544) using the combination of the spectro-interferometers AMBER (K-band) + MIDI (N-band). The closure phase capabilities of the 3-beam combiner AMBER allows to detect directly asymmetry in the dust emission on milliarcsecond scales. The object is selected as an ideal test case because the binary nature (orbital period is $499 \pm 3$ days) is well established (Maas et al. 2003) and it is bright in both wavelength bands. It is an O-rich post-AGB star of spectral type $\mathrm{F}$ with very low amplitude $V$-band variability. Its distance, based on an 
Table 1. The log of the interferometric observations of IRAS 08544. For each observation we give the physical baseline set (using VLTI-station nomenclature), the projected baseline length $(\mathrm{PB})$, the baseline angle (PA) and wavelength band ( $\lambda$-band). Note that the AMBER observations (K-band) are obtained simultaneously on a closed triangle.

\begin{tabular}{cccccc}
\hline \hline $\begin{array}{c}\text { Night } \\
\text { yyyy/mm/dd }\end{array}$ & $\begin{array}{c}\text { UT } \\
\text { hh:mm }\end{array}$ & $\begin{array}{c}\text { Baseline } \\
\text { config }\end{array}$ & $\begin{array}{c}\text { PB } \\
{[\mathrm{m}]}\end{array}$ & $\begin{array}{c}\text { PA } \\
{\left[{ }^{\circ}\right]}\end{array}$ & $\begin{array}{c}\lambda- \\
\text { band }\end{array}$ \\
\hline $2006 / 11 / 14$ & $07: 59$ & D0-G0 & 31.2 & 49.2 & $\mathrm{~N}$ \\
$2006 / 12 / 17$ & $06: 34$ & G0-H0 & 31.8 & 57.8 & $\mathrm{~N}$ \\
$2005 / 12 / 22$ & $08: 09$ & U2-U3 & 43.4 & 42.7 & $\mathrm{~K}$ \\
$2005 / 12 / 22$ & $08: 09$ & U3-U4 & 62.6 & 111.2 & $\mathrm{~K}$ \\
$2005 / 12 / 22$ & $08: 09$ & U2-U4 & 87.6 & 85.2 & $\mathrm{~K}$ \\
\hline
\end{tabular}

assumed luminosity of $5000 L_{\odot}$, is $0.8 \pm 0.2 \mathrm{kpc}$ (de Ruyter et al. 2006).

In Sect. 2 we outline the observations and the reduction process. Thereafter, we discuss the compatibility of different geometries, constrained by the spectral energy distribution (SED), with the interferometric observations. We draw our conclusions in Sect. 4.

\section{Interferometric measurements}

IRAS 08544 was observed interferometrically in both the $\mathrm{N}(8-13 \mu \mathrm{m})$ and K-band $(1.8-2.5 \mu \mathrm{m})$, with the MIDI and AMBER instrument respectively. The log of these observations is given in Table 1.

With MIDI, we observed the object at two different physical baselines with a similar projected baseline of $\sim 31 \mathrm{~m}$. The observations were performed in HIGH_SENS mode using a prism as dispersive element, resulting in a spectral resolution of $R \sim 30$. The data were reduced using the MIA+EWS reduction software (version 1.5.2). For more information on the reduction process we refer to our previous papers (Deroo et al. 2006, 2007). To calibrate the data, we used observations of Sirius obtained with the same setup and observed in concatenation with our science exposure. The resulting calibrated visibilities are shown in Fig. 2.

With AMBER (Petrov et al. 2003), we studied the K-band morphology of IRAS 08544 on the closed baseline triangle U2-U3-U4. The observations were performed in the low resolution setup $(R \sim 30)$ with an integration time of $35 \mathrm{~ms}$ per frame.

To extract the AMBER observables (i.e. 3 spectrally dispersed visibility amplitudes and a closure phase), we followed the standard procedure described in Tatulli et al. (2007). Since the observations were made in low spectral resolution, the visibilities have to be corrected for the loss of spectral coherence induced by the atmospheric differential piston. The latter was computed using the differential phase estimator as proposed in Tatulli et al. (2007). To determine the interferometric observables, we used only those frames that allow the computation of the instantaneous piston ( $\sim 70 \%$ of the total number of frames). The closure phase was determined using a "classical" bispectrum analysis (Tatulli et al. 2007; Kervella et al. 2004).

The above procedure was adopted for the calibrator (HD 78959) and the science target, after which calibrated visibility amplitudes are obtained. Note that the calibrator is essentially unresolved at the baseline triangle with a diameter of only $0.94 \pm$ 0.01 mas (Mérand et al. 2005) providing respectively $V=0.99$, $V=0.98, V=0.96$. The calibrated visibilities and phases are shown in Fig. 2. The data were limited to the spectral window $2-2.4 \mu \mathrm{m}$ because the calibrator flux outside this window was too low. To estimate the error on the obtained visibilities, we

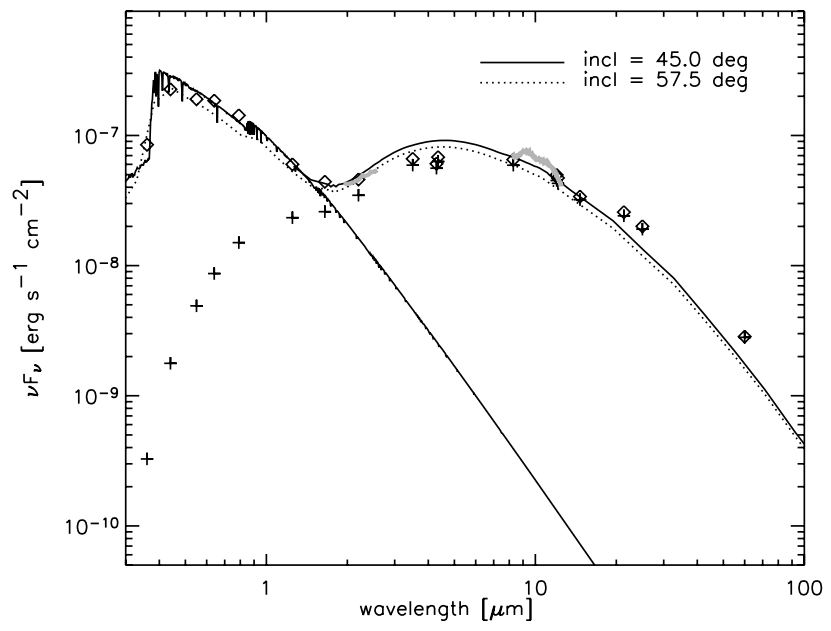

Fig. 1. The SED of IRAS 08544. The observed and dereddened $(E(B-V)=1.3)$ photometry is shown using crosses and diamonds respectively. The disc model fitted to the dereddened SED (under an inclination of $45 \mathrm{deg}$ ) is over plotted using a solid line (kurucz-model and infrared excess). Using a dotted line, we also show the SED of the passive disc model reproducing the interferometry best (inclination = $57.5 \mathrm{deg}$ ), with constraints given by the one fitted to the SED. Although not taken into account, we also present the relative AMBER and the $\mathrm{N}$-band (TIMMI) spectrum in grey.

took the typical AMBER error of $6 \%$ on the amplitudes. The error on the closure phases was obtained using the standard deviation of the closure phase of the calibrator.

\section{Modelling}

In Fig. 1, we reproduce the SED, with the data given in Maas et al. (2003). IRAS 08544 shows a clear infrared excess originating from a circumstellar environment containing both hot and cold dust. The total reddening, assuming the interstellar extinction law, is $E(B-V)=1.3 \pm 0.1$ of which the interstellar medium contributes at least 0.7 . This is derived from the saturated DIBs (Maas et al. 2003).

Maas et al. (2003) proposed that the dust is confined in a stable disc instead of a spherical outflow. The problem of determining the different dust geometries based on the broad band SED is degenerate. We took the SED as a starting point to determine the relevant parameters in both a spherical outflow as well as a disc, after which we confront the models with the interferometric observations.

\subsection{A spherical shell model}

Spherical dust shell models were computed with MODUST (Bouwman et al. 2000), in which the shell and dust parameters are strongly constrained by the SED: we require either amorphous carbon or very large dust grains $(10-300 \mu \mathrm{m}$ with $\alpha=3.5)$ to generate the observed large excess with little circumstellar extinction $(E(B-V)<0.6)$. We find the inner radius of this dust to be $\sim 30 R_{\star}$. Although the broad band SED can be reproduced well with this model, it is far too extended to be compatible with the interferometry: the simulated AMBER observations show almost exclusively correlated flux from the central star and as a consequence the only possible origin of asymmetry, the offset of the primary star in its binary orbit, produces closure phases $\left(\mathrm{CP}_{\text {model }}<5 \mathrm{deg}\right)$ an order of magnitude smaller than observed. Also, the simulated MIDI visibilities are too low 
by a factor of 2 . There is no possibility to match a more compact shell to the observed SED, which leads us to the clear conclusion that a spherical dust shell cannot reproduce the observations.

\subsection{A disc model}

Compliant with the energetics of the source, we assume a passive (i.e. non-accreting) disc, consisting of a mixture of gas and dust, with a large inner hole. This hole occurs because dust cannot exist above sublimation temperature $(\sim 1500 \mathrm{~K})$. The main consequence of this inner hole, is the occurrence of a vertical boundary irradiated directly by the stellar flux. Indeed, the resulting high temperature of this region will cause this inner disc rim to be puffed up by gas pressure. Depending of the optical depth, this could result in the shadowing of the material directly behind this inner rim, leading to a decrease in disc scale height. At larger distances, the disc height may be large enough again and emerge from the shadow.

We used a 2D radiative transfer code developed by Dullemond (2002, and further updates) to compute the temperature and vertical density structure of the disc. For a given structure, the temperature is determined by radiative transfer through a Monte Carlo method, which is then used to determine the density structure based on the equations of hydrostatic equilibrium. This procedure is iterated to obtain the full disc structure. For more details on this code, we refer to Dullemond (2002) and Dullemond \& Dominik (2004).

As a first step, we determined a model reproducing the broad band SED of IRAS 08544. We limited the parameters to be fitted to the SED as (1) the inner radius of the disc and (2) the total amount of mass in the disc. For the other parameters involved, we took representative values for the group of binary post-AGB objects, discussed below.

The primary was taken to be a $5000 L_{\odot}$ giant with a spectroscopically determined temperature of $7250 \mathrm{~K}$. The secondary is ignored since it is too faint. The mass of the central binary was taken as $1 M_{\odot}$ (i.e. $0.6 M_{\odot}$ for the primary, $0.4 M_{\odot}$ for the secondary, in line with the observed mass function, Maas et al. 2003). The dust input parameters are based on the constraints from the larger sample where high crystallinity fractions and large grains, up to $850 \mu \mathrm{m}$, are observed (de Ruyter et al. 2005). We took the fraction of crystalline particles (i.e. forsterite) as $50 \%$ and represented the grain size distribution from 0.1 to 850 micron, as a power law with power index -3 (i.e. the mean value suggested for circumstellar discs in D'Alessio et al. 2001). The outer radius and the density distribution of the dusty disc were taken to be the same as for the gas distribution in the Keplerian disc of the Red Rectangle (as measured by $\mathrm{CO}$ lines in Bujarrabal et al. 2005): The outer radius was set at $560 \mathrm{AU}$ and the surface density distribution was presented by a power law with index -2 .

With these parameters, we fitted the inner radius and total mass of the disc to the dereddened SED. For this initial SED fit, we assumed an inclination of $45 \mathrm{deg}$. We find a best fit for an inner radius of $9 \mathrm{AU}$ and a total mass of the circumstellar environment of $0.015 M_{\odot}$. The resulting fit to the broad band SED is shown in Fig. 1. The disc model consists of a large puffed up inner rim with scale height $\mathrm{H} / \mathrm{R}=0.22$ (total height $=2 \mathrm{H}=$ 4.0 AU) at the inner radius. The dust environment is shadowed from direct stellar light by this large puffed up inner rim until 70 AU. From 70 AU onwards, the disc emerges from the shadow and reaches a scale height of 0.43 at the outer radius.

The fit of our disc model to the SED determines almost all parameters relevant to the visibility fit: the distance of the
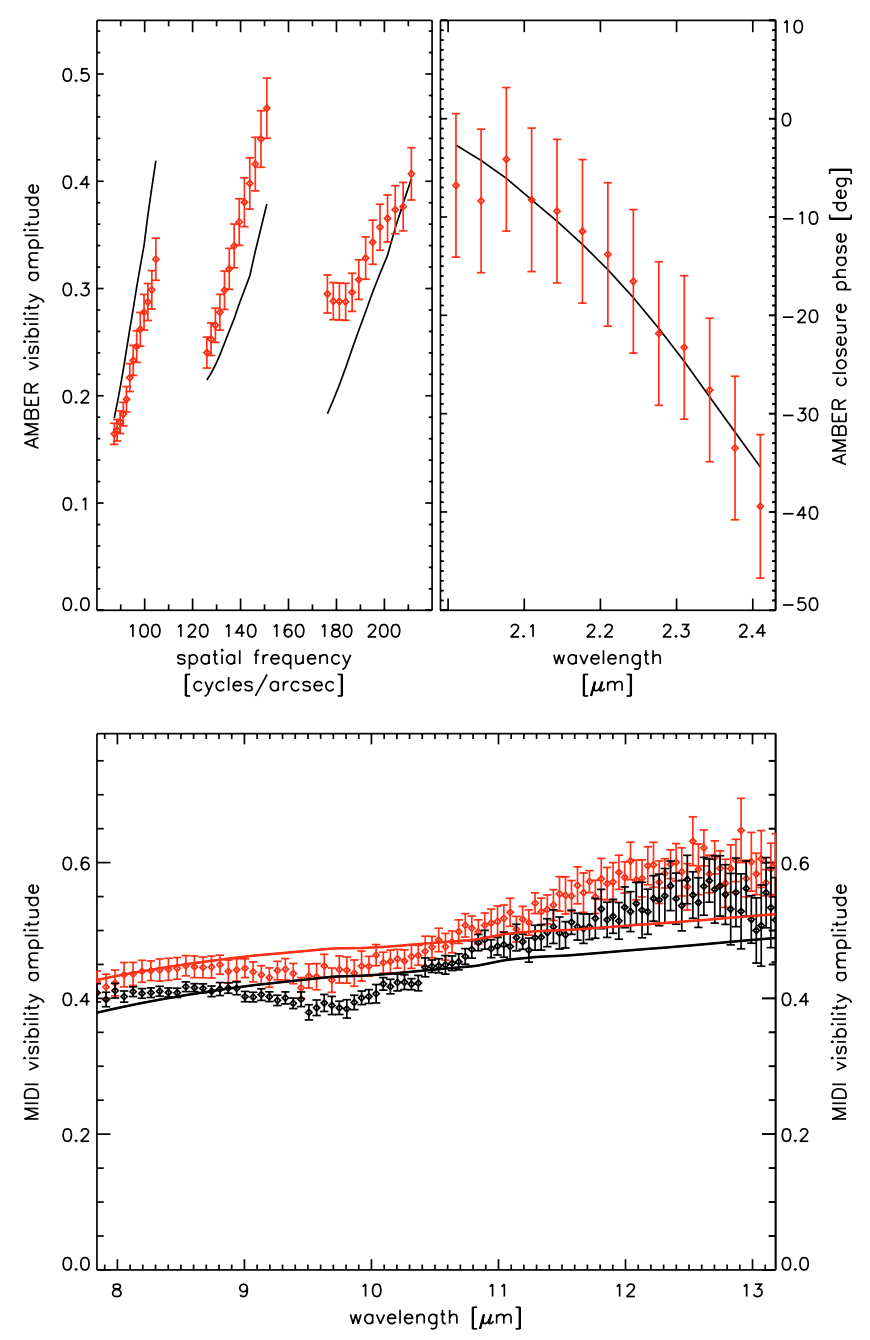

Fig. 2. Top panel: a comparison between the observed (diamonds with error bars) and the modelled (solid line) visibility amplitudes (left) and closure phase (right) in the K-band. Bottom panel: the same but now for the N-band. Different colours are used for both measurements: red for 2006/11/14 and black for 2006/12/17. The physical model, which was constrained only by the SED, predicts the size of the emission in both wavelength regimes, as well as the asymmetrical nature in the K-band.

system, the angular scale of the inner radius, the scale height of the disc etc. The only free parameters are now: (1) the inclination of the disc and (2) the orientation of the inclined disc in the plane of the sky. Note that inclinations producing circumstellar extinction in the line of sight cannot be excluded, since part of the reddening could be circumstellar (see above).

For a large set of inclinations, with a step of $\Delta=2.5 \mathrm{deg}$, we made images which we rotated under different orientation angles $(\Delta=1 \mathrm{deg})$ on the sky. For every grid point we reproduced the interferometric observables after which we compare them to all observations ( $\mathrm{N}$ and $\mathrm{K}$ amplitudes and $\mathrm{K}$ closure phase). We find the minimal $\chi^{2}=4.8$ for an inclination of $58_{-30}^{+5} \mathrm{deg}$ and an orientation of $186 \pm 10 \mathrm{deg}$ East of North. With the current uv-coverage, the inclination of the orbit is not too well constrained which is reflected by the large error bars. The resulting images at $2 \mu \mathrm{m}$ and $10 \mu \mathrm{m}$ for the best fit are shown in Fig. 3. The comparison between the measured and modelled visibilities is shown in Fig. 2. The agreement between the interferometric data and the model is very good, despite the fact that we only fit the inclination and orientation of the disc to the 

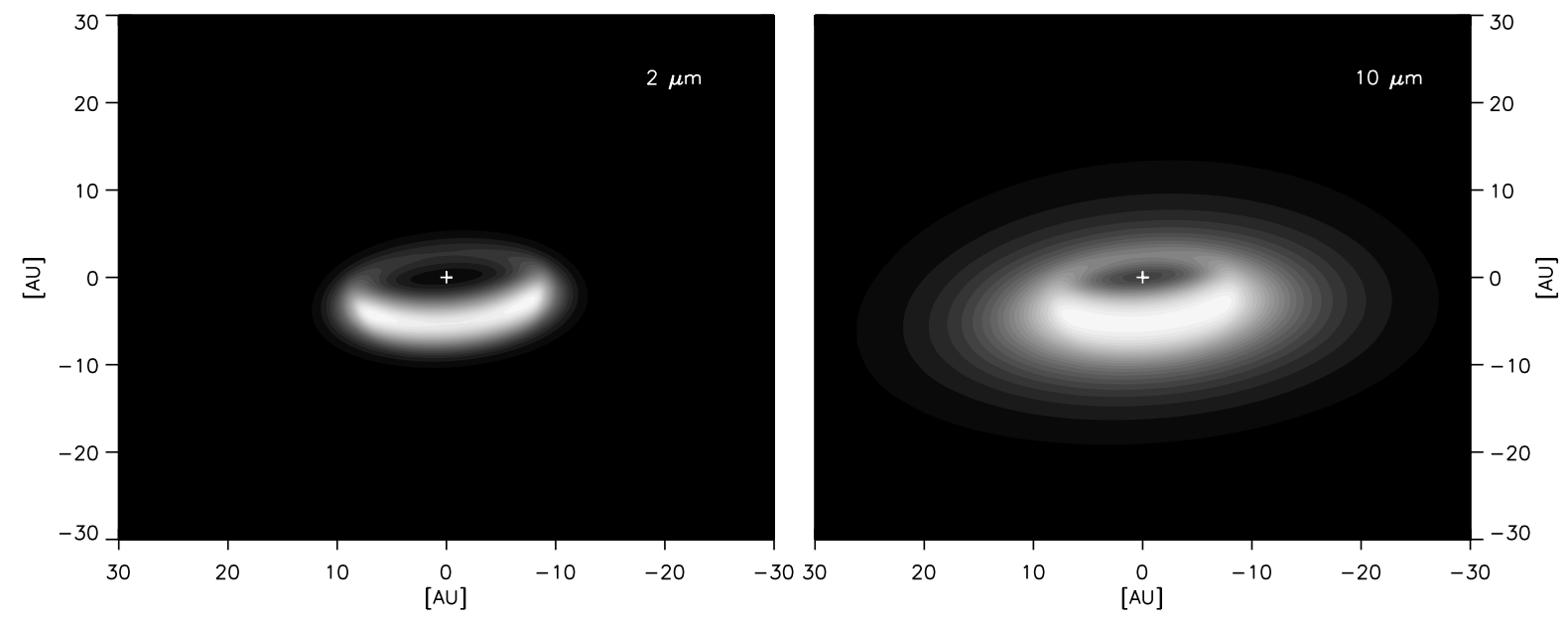

Fig. 3. The morphology of the circumstellar environment of IRAS 08544 in the K-band ( $2 \mu \mathrm{m}$, left panel) and the N-band (10 $\mu \mathrm{m}$, right panel): North is up and East is to the left. The position angles of the baselines in the K-band are 43, 85 and 112 deg and in the N-band 49 and 58 deg. The images are contour plots using 25 equally spaced levels which are filled in greyscale. The position of the giant is indicated by the cross. The brightest region is the inner wall on the far side.

interferometric observables. The disc model was entirely constrained by the SED and it agrees with the spatially resolved measurements both in the $\mathrm{K}$ and $\mathrm{N}$-band. The interferometric observables show that our physical model of a disc structure with a large puffed-up inner rim, is correct. However, it is clear that there is still room for some fine-tuning which will require a better uv-coverage. The discrepancy for the MIDI visibilities around $9.8 \mu \mathrm{m}$ is related to the spectral features of amorphous silicates, which is natural since we chose to focus on global geometry and not specific spectral features. Moreover, the observed slope of the MIDI visibility curves are very steep showing that the intensity distribution is very compact even at $13 \mu \mathrm{m}$. This could be linked to an even steeper density distribution or a disc which is more compact.

\section{Discussion and conclusions}

The combination of MIDI and AMBER interferometric measurements is a powerful tool to study the geometry of the circumstellar material around binary post-AGB stars.

We could easily eliminate a spherical model. Assuming a passive irradiated dusty disc in equilibrium instead, we constrained a self consistent model of the circumstellar material around IRAS 08544 which fits the observed SED. The interferometric test of the model is very constraining: the size in both wavelength regimes must be accurately determined, as well as the asymmetry (the non-zero closure phase). Moreover, the morphology change between both wavelength regimes, related to the temperature distribution, must be reproduced. Clearly all of these requirements are met with our disc model.

We therefore argue that we presented direct evidence for a stable disc around IRAS 08544. The SED, the non-zero closure phase and the visibilities show that this disc is circumbinary with a large puffed up inner rim.

This object was selected as a prototype of a larger sample of binary post-AGB objects all displaying evidence for a stable Keplerian disc. All orbits determined so far (e.g. Van Winckel 2007, and references therein) are too small to accommodate a full grown AGB star. The objects must have been subject to severe binary interaction during their evolution. We postulate that our result of this particular object may be representative for the whole group of binaries. Note that only for one binary of the sample (HD 44179 and its Red Rectangle nebula) the Keplerian rotation has been directly detected (Bujarrabal et al. 2005).

Dust grains in stable discs are subject to several physical processes like efficient annealing, grain growth, gas-grain chemical interactions etc., very much like the dust evolution in protoplanetary discs around young stellar objects. Refinements of the disc model presented here will need to include these effects, especially if we want to study the evolution of these "protoplanetary" discs in the rapidly changing radiation field of the central evolved star. This will need in depth (interferometric) studies of a larger sample of objects as well as theoretical studies on the formation and evolution of the discs which play a lead role in the final evolution of the presented binaries.

Acknowledgements. We would like to thank Kees Dullemond for providing his 2D radiative transfer code. P. D. acknowledges FWO support under contract G.0178.02.

\section{References}

Balick, B., \& Frank, A. 2002, ARA\&A, 40, 439

Bouwman, J., de Koter, A., van den Ancker, M. E., \& Waters, L. B. F. M. 2000, A\&A, 360, 213

Bujarrabal, V., Castro-Carrizo, A., Alcolea, J., \& Neri, R. 2005, A\&A, 441, 1031 D’Alessio, P., Calvet, N., \& Hartmann, L. 2001, ApJ, 553, 321

de Ruyter, S., van Winckel, H., Dominik, C., Waters, L. B. F. M., \& Dejonghe, H. 2005, A\&A, 435, 161

de Ruyter, S., van Winckel, H., Maas, T., et al. 2006, A\&A, 448, 641

Deroo, P., van Winckel, H., Min, M., et al. 2006, A\&A, 450, 181

Deroo, P., van Winckel, H., Verhoelst, T., et al. 2007, A\&A, 467, 1093

Dullemond, C. P. 2002, A\&A, 395, 853

Dullemond, C. P., \& Dominik, C. 2004, A\&A, 417, 159

Kervella, P., Ségransan, D., \& Coudé du Foresto, V. 2004, A\&A, 425, 1161

Maas, T., Van Winckel, H., Lloyd Evans, T., et al. 2003, A\&A, 405, 271

Mérand, A., Bordé, P., \& Coudé Du Foresto, V. 2005, A\&A, 433, 1155

Moe, M., \& De Marco, O. 2006, ApJ, 650, 916

Petrov, R. G., Malbet, F., Weigelt, G., et al. 2003, in Interferometry for Optical Astronomy II, ed. W. A. Traub, Proc. SPIE, 4838, 924

Sahai, R., Morris, M., Sánchez Contreras, C., \& Claussen, M. 2007, ApJ, in prep. Tatulli, E., Millour, F., Chelli, A., et al. 2007, A\&A, 464, 29

Van Winckel, H. 2003, ARA\&A, 41, 391

Van Winckel, H. 2007, Baltic Astron., 16, 112

Zijlstra, A. A. 2007, Baltic Astron., 16, 79 